\title{
Evaluation of additional head of biceps brachii: a study with autopsy material
}

\author{
L.E. Ballesteros, P.L. Forero, E.R. Buitrago \\ Industrial University of Santander, Bucaramanga, Colombia \\ [Received 20 September 2013; Accepted 5 November 2013]
}

\begin{abstract}
Additional head of the biceps brachii (AHBB) has been reported in different population groups with a frequency of $1-25 \%$. The purpose of this study was to determine the incidence and morphologic expression of the AHBB as determined in a sample of the Colombian population. An exploration was conducted with 106 arms corresponding to unclaimed corpses autopsied at Institute of Legal and Forensic Medicine of Bucaramanga, Colombia. Using medial incision involving skin, subcutaneous tissue, and brachial fascia, the heads of the biceps and their innervating branches were visualised. One AHBB was observed in 21 (19.8\%) of the arms evaluated, with non-significant difference $(p=0.568)$ per side of presentation: 11 (52.4\%) cases on the right side and 10 (47.6\%) on the left side. All AHBBs were originated in the infero-medial segment of the humerus, with a mean thickness of $17.8 \pm 6.8 \mathrm{~mm}$. In $4(19 \%)$ cases the fascicle was thin, less than $10 \mathrm{~mm}$; in $7(33.3 \%)$ cases it was of medium thickness, between 11 and $20 \mathrm{~mm}$, whereas in $47.6 \%$ it was longer than $20 \mathrm{~mm}$. The length of the $A H B B$ was $118.3 \pm 26.8 \mathrm{~mm}$; its motor point supplied by the musculocutaneous nerve was located at $101.3 \pm 20.9 \mathrm{~mm}$ of the bi-epicondylar line. The incidence of AHBB in this study is located at the upper segment of what has been reported in the literature and could be a morphologic trait of the Colombian population; in agreement with prior studies, the origin was the infero-medial surface of the humerus. (Folia Morphol 2014; 73, 2: 193-198)
\end{abstract}

Key words: arm, biceps brachii muscle, additional head of biceps brachii, musculocutaneous nerve

\section{INTRODUCTION}

The biceps brachii muscle (BB), located in the anterior compartment of the arm, has usually 2 heads - the short one originates from the coracoid process and the long one stems from the supraglenoid tubercle. Two heads join at the juncture of the upper and mid third of the forearm and then its tendon appears in the proximity of the bi-epicondylar line, and is inserted in the radial tuberosity. It contributes to the flexion and supination of the forearm and is innervated by the musculocutaneous nerve (MCN) $[4,5]$.

The $\mathrm{BB}$ is a muscle with large variability due to the presence of supernumerary heads arising from the deltoid and pectoralis major muscles, the articular capsule of the shoulder, the coracoid process, the intertubercular sulcus, and the mid third of the humerus, its most common variation $[5,8,9,11$, 24]. Presence of additional heads of the biceps bra-

Address for correspondence: Prof. L.E. Ballesteros, Departamento de Ciencias Básicas, Facultad de Salud — Universidad Industrial de Santander, Cra 32 \# 29-31, 680003 Bucaramanga, Colombia, tel/fax: +57 (7) 6455693, e-mail: Iballest56@yahoo.es 
chii (AHBB) has been reported in a range of $1-25 \%$ with its unilateral occurrence being more frequent than its bilateral occurrence $[2,11,14,17-20]$. The AHBB has functional and clinical implications given by the possibility of enhancing typical functions to the muscle or producing symptoms of neurovascular compression $[7,17,26]$. Knowing it well allows for the evaluation and management of the disorders of the upper extremity in situations involving these muscular structures. Moreover, this knowledge is relevant for planning surgical approaches in the arm $[5,7,20]$.

Determination of the characteristics of the AHBB has been described with classic dissection techniques or imaging studies in other population groups, some with a clear basic-clinical orientation $[3,6,8,11$, $16,22]$. Taking into account that the ethnic factor is decisive for the presence of different anatomic expressions of the AHBB and the sparse information of this type in the Mestizo population, the predominant ethnic group in Latin America, it is appropriate to perform this study in fresh cadaver material in order to obtain our own reference information to enrich the morphologic concepts and the medical and surgical applications.

\section{MATERIALS AND METHODS}

This non-probabilistic cross-over descriptive study determined the frequency and morphologic characteristics of the AHBB in 106 arms corresponding to 53 fresh cadaver specimens, autopsied at National Institute of Legal and Forensic Medicine of Bucaramanga, Colombia. The sample met the following inclusion criteria: adult males pertaining to the Mestizo racial group (a breed of white Hispanics and natives), without evidence of direct trauma or pathologies involving upper extremity and who were not being subjected to forensic investigation.

An extensive approach of the anterior compartment of the arm was performed using a midline incision, from the mid third of the clavicle to the crease of the elbow, lowering the medial and lateral fasciocutaneous flaps; the muscle pectoralis major was released from its clavicular and sternal origins, thus allowing to visualise the terminal branches of the brachial plexus in the axillary region. Then, the heads of the BB were dissected and the nerve branches that innervate it from its origins to the respective motor points were isolated. The existence of AHBB was recorded along with its morphologic characteristics, rating its thickness as thin when it was less than $10 \mathrm{~mm}$; medium, between 11 and $19 \mathrm{~mm}$, and thick when the thickness was equal to or greater than $20 \mathrm{~mm}$. Similarly, the length of the additional head was measured. According to the criteria of Rodriguez-Niedenfuhr et al. [20], the location of the AHBB was typified as infero-medial, superior and infero-lateral.

All morphometric assessments were made with a digital caliber (Mitotuyo ${ }^{\circledR}$ ), and the findings were photographed with Canon T2I camera. The findings were digitised in Excel tables and statistical analyses were conducted using Epi-Info 3.5.3. Nominal variables were described with ratios, and continuous variables with means and standard deviations. Statistical tests included $\chi^{2}$ and Student's $t$ test accepting an alpha error of up to $5 \%$.

\section{RESULTS}

One AHBB was observed in 21 (19.8\%) of the arms evaluated, with no significant differences ( $p=0.568$ ) per side of presentation: 11 cases on the right side (52.4\%) and $10(47.6 \%)$ on the left side. There was a bilateral occurrence in $7(50 \%)$ of all cadaver specimens.

In all cases, the AHBB was located in the inferomedial segment of the arm, originating in the surface of the humerus adjacent to the insertion of the coracobrachialis muscle and closely related to the internal intermuscular septum of the arm. The muscle proceeded inferiorly and deeply with respect to the short head of the BB and of the brachialis muscle; it ended up fusing itself to the tendon of the $\mathrm{BB}$, next to the bi-epicondylar line.

The length of the AHBB was $118.3 \pm 26.8 \mathrm{~mm}$ and the width measured at the mid segment was $17.8 \pm$ $\pm 6.8 \mathrm{~mm}$, corresponding to $15.1 \pm 7 \mathrm{~mm}$ to the left side and $20.3 \pm 8.8 \mathrm{~mm}$ to the right side, with this difference being statistically significant $(p=0.035)$. In $4(19 \%)$ cases, the muscular fascicle was thin (Fig. 1); it was medium in 7 (33.3\%) (Fig. 2), and thick in $10(47.6 \%)$ specimens (Fig. 3).

In all cases the MCN innervated the AHBB. In 1 case, the MCN shared innervation with a communicating branch originating from the median nerve (Fig. 4). In 12 (57.1\%) specimens, the innervating branch stemmed from the MCN before the emergence of the branch for the brachialis muscle (Fig. 5); in $9(42.9 \%)$ cases, the innervating branch for the AHBB emerged from the branch for the brachialis muscle. The length of the innervating branch was 


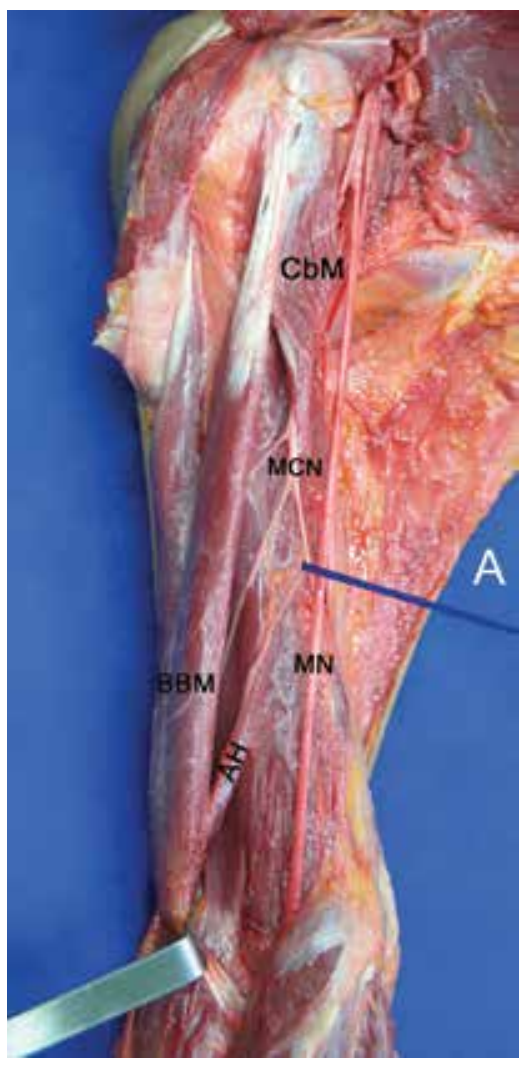

Figure 1. Medial view of the right arm; A - axilla; BBM — biceps brachii muscle; $\mathrm{AH}$ — additional head of biceps brachii (thin); $\mathrm{CbM}$ - coracobrachialis muscle; $\mathrm{MCN}$ - musculocutaneous nerve; $\mathrm{MN}$ - median nerve.

$36.1 \pm 12.3 \mathrm{~mm}$, and the motor point was located at $101.3 \pm 20.9 \mathrm{~mm}$ of the bi-epicondylar line.

In $5(23.8 \%)$ cases, the AHBB showed the presence of the communicating branch between the MCN and the median nerve, and in $2(9.5 \%)$ cases it had an anomalous origin from the MCN characterised in that it did not perforate the coracobrachialis muscle (Fig. 5).

\section{DISCUSSION}

The presence of the AHBB is an uncommon variation in some population groups. Low incidences in a range of $0.7-3.8 \%$ have been reported by several studies $[5,6,10,11,16-18,21,22]$. Santos-Neto et al. [23] and other investigators report mean incidences within a range of $6.2-12.5 \%[3,7,9,13,17,20,27]$. Our findings (19.8\%) are in the high incidence reported by Al-Kushi [2] - 15\%, Santo-Neto et al. [23] in a Caucasian population - 20\%, Asvat et al. [3] $21 \%$ and Rincon et al. [19] $-25 \%$.

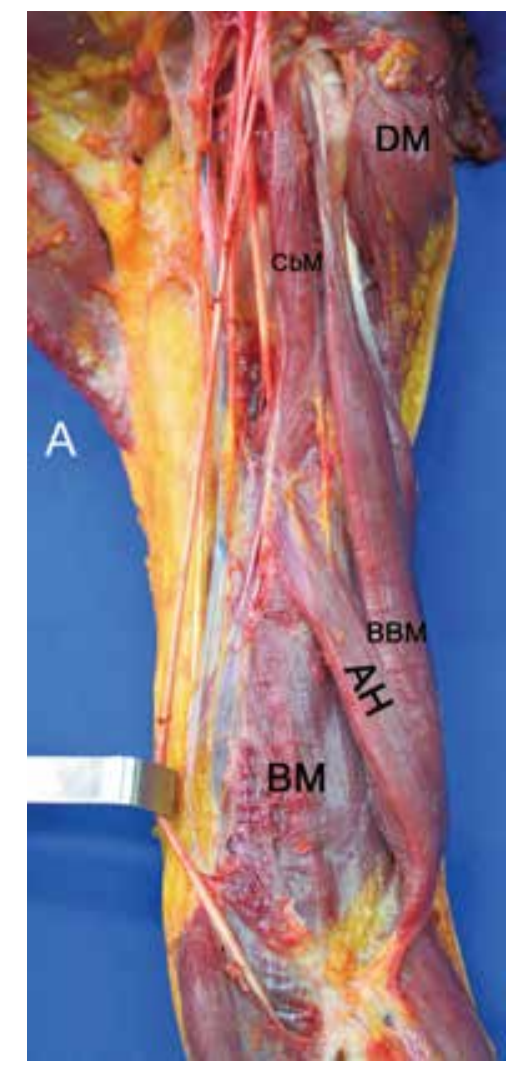

Figure 2. Medial view of the left arm; A - axilla; BBM — biceps brachii muscle; $\mathrm{AH}$ - additional head of biceps brachii (medium); $\mathrm{CbM}$ - coracobrachialis muscle; $\mathrm{BM}$ - brachial muscle; $\mathrm{DM}$ deltoid muscle.

The unilateral and predominantly right-sided occurrence is reported by diverse authors as the most frequent one [2, 17-20], whereas Asvat et al. [3], Kumar et al. [10] and Valekar et al. [27] reported high prevalence of the bilateral expression. In our series we have found a balance between the bilateral and unilateral expressions, in agreement with other studies [13, 22].

The wide range of incidence of AHBB reported by diverse authors is probably due to the ancestral biologic characteristics that determine the variable expression of these structures in the population groups evaluated. Thus, for example, different studies conducted in the Indian population have reported a low incidence of the AHBB $[5,10,16,18,22]$, whereas reports from Caucasian populations are controversial: Khaledpour [6] report a low incidence, whereas Santo-Neto et al. [23] report $20 \%$ incidence. Our study conducted in the Mestizo population reports a high incidence, but slightly lower than the incidence 


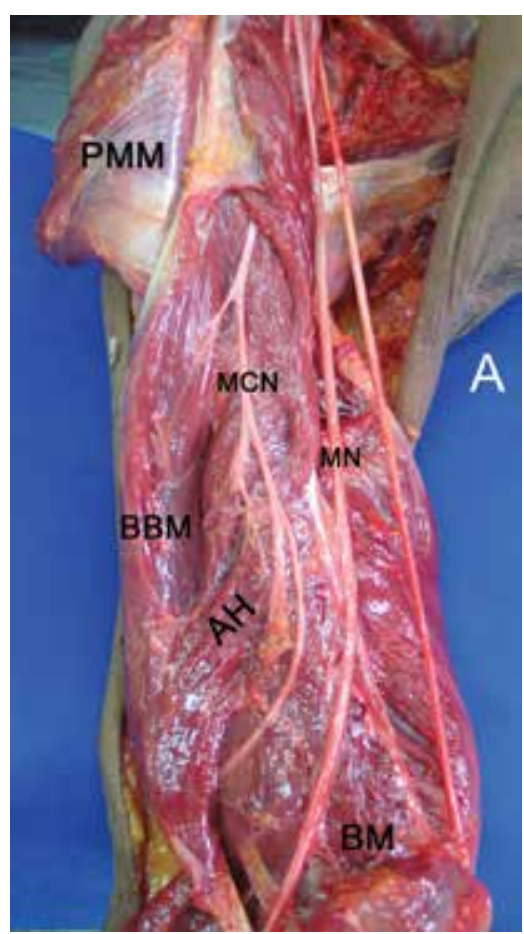

Figure 3. Medial view of the right arm; A - axilla; PMM - pectoralis major muscle sectioned; BBM — biceps brachii muscle; $\mathrm{AH}$ - additional head of biceps brachii (thick); $\mathrm{BM}$ - brachialis muscle; MCN — musculocutaneous nerve; $\mathrm{MN}$ — median nerve.

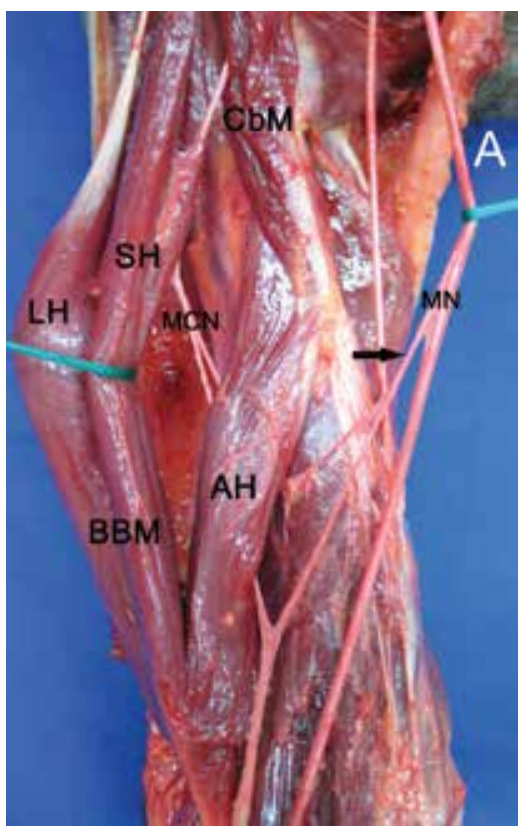

Figure 4. Medial view of the right arm; A — axilla; $\mathrm{SH}$ - small head of biceps brachii; $\mathrm{LH}$ - large head of biceps brachii; BBM — biceps brachii muscle; AH — additional head of biceps brachii (thick sized); $\mathrm{CbM}$ - coracobrachialis muscle; $\mathrm{MCN}$ - musculocutaneous nerve; $\mathrm{MN}$ — median nerve; Arrow — communicating MN-MCN branch.

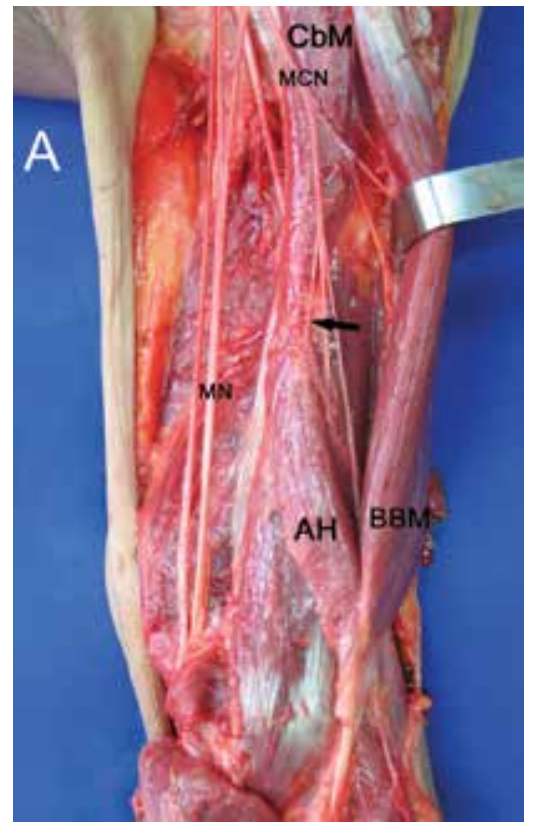

Figure 5. Medial view of the left arm; A - axilla; BBM — biceps brachii muscle; $\mathrm{AH}$ - additional head of biceps brachii; $\mathrm{CbM}$ coracobrachialis muscle; MCN — musculocutaneous nerve (without perforating coracobrachialis muscle); $\mathrm{MN}$ - median nerve; Arrow - branch of the MCN to the AH.

reported in this same group population by Rincón et al. [19] (25\%). Similarly, it should be taken into account that sample size has a substantial impact on the figures reported, and therefore reports from studies with small samples are unreliable, thus allowing us to infer that as the universe of the structures evaluated grows larger, the figures reported will vary substantially $[3,11,19,20]$. The presence of AHBB may be clinically silent and go unnoticed until it is discovered during a surgical procedure or an autopsy. This variation acquires special meaning during surgical procedures of the inferior segment of the arm and the elbow, because it may cause difficulties for the operator to appropriately recognise the structures composing this anatomic region, thus increasing the risk of iatrogenesis $[5,17,18]$.

Similar to what was observed in our study, the infero-medial surface of the humerus has been reported in the literature as the most frequent site of origin of the AHBB $[3,5,7,11,13,17,20]$. Few works $[10,14,27]$ have reported the predominant localisation of the AHBB in the upper segment of the arm, joined to its long or short heads. Asvat et al. [3] and 
Rodriguez-Niedenfuhr et al. [20] have reported the anterolateral segment of the arm as a rare location for the AHBB.

The supernumerary head originating in the anteromedial surface of the humerus, adjacent to the insertion zone of the coracobrachialis muscle, may be interpreted as representing a remanent of the long portion of the coracobrachialis muscle, a condition observed in ancestral hominidae, where the coracobrachialis is inserted in the radial tuberosity sharing a tendon with the $\mathrm{BB}$; it has also been proposed, that the AHBB is a derivation of the brachialis muscle whose insertion has been translocated from the coracoid process of the ulna to the radial tuberosity $[18,25]$. Similarly, modifications in mesenchymal condensation during embryogenesis characterised by contact between muscle and nerve cells of the upper extremity, probably determined by gene factors, could explain the presence of this anatomic variation [15].

Few of the prior works have been focused on the morphometric aspects of the AHBB. The length of the additional head found in the present study $(118.3 \mathrm{~mm})$ is similar to that reported by Rincón et al. [19], llayperuma et al. [11] and some other authors $[4,9]$ whereas Parasquevas et al. [16] and Abu-Hijleh et al. [1] reported a length of $65 \mathrm{~mm}$ and $95 \mathrm{~mm}$, respectively. The width of this muscle head has been reported within a range of 15-24 mm $[1,5,9,11,16,19,27]$, consistent with our findings. The characterisation of the size of the AHBB as thin, medium and thick, proposed in the present study, allows to enrich the descriptions of this structure. Of note, a thick AHBB was found with the higher frequency $(47.6 \%)$ in this work. Most prior reports $[1,3,5,11,19-21]$ coincide in indicating that the AHBB is innervated by the MCN. We have found 1 case of shared innervation between the MCN and the communicating branch of the median nerve. Al-Kushi [2] has reported the innervation of the AHBB by the median nerve, very likely in the absence of the MCN. Similarly, no prior work has ever made a morphometric and qualitative characterisation of the innervation of the AHBB, this information being relevant for arm surgery planning.

The presence of the AHBB has both functional and clinical implications; it may contribute to an increase in the flexion and supination capacity of the forearm, and when the presence is unilateral, it is often confused with some soft tissue tumour. In presence of fractures of the segment distal of the humerus, it contributes to or increases bone displacement $[9,13,17,23]$.

The AHBB associated with the simultaneous presence of the communicating branch between the musculocutaneous and median nerves observed in our series in $23.8 \%$ of the cases, is a characteristic also described in the works by Kosugi et al. [9] and Maeda et al. [12], who reported this trait in $27.8 \%$ and $30.1 \%$, respectively, in Japanese populations. Having in mind the presence and location of the communicating branch in the mid or lower thirds of the arm associated to muscle variants, such as the AHBB during surgical approaches, will possibly decrease the risk of injuring these structures. An injury of the musculocutaneous nerve above the emergence of the communicating branch may cause the paralysis of the supernumerary head of the biceps brachii, of the brachialis muscle, and anaesthesia of the lateral aspect of the forearm, in addition to the partial motor and sensorial deficit expected from the injury of the median nerve.

\section{CONCLUSIONS}

The incidence of AHBB observed in our work is in the upper limit of the range reported in the literature, and could be a morphologic trait of the Colombian population. In agreement with prior studies, in most cases this structure originates from the infero-medial surface of the humerus. Of note is the significant simultaneous presence of the AHBB with an anomalous origin, and branches communicating the MCN to the median nerve. Morphologic characterisation of the AHBB and the morphometry of its innervation provide relevant information for surgical planning of the distal segment of the arm.

\section{REFERENCES}

1. Abu-Hijleh MF (2005) Three-headed biceps brachii muscle associated with duplicated musculocutaneous nerve. Clin Anat, 18: 376-379.

2. Al-Kushi AG (2013) Anatomical study of the third head of biceps brachii muscle and its innervation by median nerve in human dissection. J Clin Med Res, 5: 47-52.

3. Asvat R, Candler P, Sarmiento EE (1993) High incidence of third head of biceps brachii in South African populations. J Anat, 182: 101-104.

4. Bergman RA, Thompson SA, Afifi AK, Saadeh FA (1988) Compendium of human anatomic variations. Urban \& Schwarzenberg, Baltimore, pp. 139-143.

5. Cheema P, Singla R (2011) Low incidence of the third head of the biceps brachii in the North Indian population. J Clin Diagn Res, 5: 1323-1326. 
6. Khaledpour C (1985) Anomalies of the biceps muscle of the arm. Anat Anz, 158: 79-85.

7. Kervancioglu P, Orhan M (2011) An anatomical study on the three-headed biceps brachii in human fetuses and clinical relevance. Folia Morphol, 70: 116-120.

8. Kopuz C, Sancak B, Ozbenli S (1999) On the incidence of third head of biceps brachii in Turkish neonates and adults. Kaibogaku Zasshi, 74: 301-305.

9. Kosugi K, Shibata S, Yamashita H (1992) Supernumerary humeral heads of the biceps brachii and branching pattern of the musclocutaneous nerve in Japanese. Surg Radiol Anat, 14: 175-185.

10. Kumar H, Das S, Rath G (2008) An anatomical insight into the third head of biceps brachii muscle. Bratisl Lek Listy, 109: 76-78.

11. Ilayperuma I, Nanayakkara G, Palahepitiya, N (2011) Incidence of humeral head of biceps brachii muscle: anatomical insight. Int J Morphol, 29: 221-225.

12. Maeda S, Kawai K, Koizumi M, Ide J, Tokiyoshi A, Mizuta H, Kodama K (2009) Morphological study of the communication between the musculocutaneous and median nerves. Anat Sci Int, 84: 34-40.

13. Nakatani T, Tanaka S, Mizukami S (1998) Bilateral fourheaded biceps brachii muscles: The median nerve and the brachial artery passing through a tunnel which is formed by a muscle slip from the accessory head. Clin Anat, 11: 209-212.

14. Nayak SR, Prabhu LV, Sivanandan R (2006) Third head of biceps brachii: a rare occurrence in the Indian population. Ann Anat, 188: 159-161.

15. Pacholczak R, Klimek-Piotrowska W, Walocha, JA (2011) Absence of the musculocutaneous nerve associated with a supernumerary head of biceps brachii: a case report. Surg Radiol Anat, 33: 551-554.

16. Paraskevas $G$, Natsis $K$, loannidis $O$, Papaziogas $B$, Kitsoulis $P$, Spanidou $S$ (2008) Accessory muscles in the lower part of the anterior compartment of the arm that may entrap neurovascular elements. Clin Anat, 21: 246-251.

17. Poudel PP, Bhattarai C (2009) Study on the supernumerary heads of biceps brachii muscle in Nepalese. Nepal Med Coll J, 11: 96-98.

18. Rai R, Ranade AV, Prabhu LV, Pai MM, Prakash (2007) The third head of the biceps brachii in the Indian population. Singapore Med J, 48: 929.

19. Rincón F, Rodríquez ZJ, Sánchez A, Léon A, González IF (2002) The anatomic characteristics of the third head of biceps brachii muscle in Colombian population. Rev Chil Anat, 20: 197-200.

20. Rodríguez-Niedenführ M, Vázquez T, Choi D, Parkin I, Sañudo JR (2003) Supernumerary humeral heads of the biceps brachii muscle revisited. Clin Anat, 16: 197-203.

21. Rodríguez-Vázquez JF, Mérida-Velasco JR, Jiménez-Collado J (1999) Unusual variation of a third head of the biceps brachii muscle, Ann Anat, 181: 573-575.

22. Sandeep P, Bharat B, Amrut M (2012) A study on the accessory head of the biceps brachii in Indians which was done during cadaver dissections, J Clin Diag Res, 6: 1137-1139.

23. Santo-Neto H, Camilli JA, Andrade JC, Meciano Filho J, Marques MJ (1998) The incidence of the biceps brachii third head in Brazilian white and blacks, Ann Anat, 180: 69-71.

24. Sargon MF, Tuncali D, Celik HH (1996) An unusual origin for the supernumerary head of biceps brachii muscle. Clin Anat, 9: 160-162.

25. Sonntag CF (1924) On the anatomy, physiology and pathology of the orang-outang. Proceedings of the Zoological Society, 94: 349-450.

26. Warner JJ, Paletta GA, Warren RF (1992) Accessory head of the biceps brachii: case report demonstrating clinical relevance. Clin Orthop, 280: 179-181.

27. Varlekar P, Meghatar NK, Mehta CD (2011) Incidence of the third head of biceps brachii in Western Indian population. NJIRM, 2: 65-67. 